\title{
Soluble recombinant influenza vaccines
}

\author{
Walter Fiers*, Sabine Neirynck, Tom Deroo, Xavier Saelens and Willy Min Jou \\ Laboratory of Molecular Biology, University of Ghent and Flanders Interuniversity Institute for Biotechnology, \\ K. L. Ledeganckstraat 35, B-9000 Ghent, Belgium
}

\begin{abstract}
Soluble, recombinant forms of influenza A virus haemagglutinin and neuraminidase have been produced in cells of lower eukaryotes, and shown in a mouse model to induce complete protective immunity against a lethal virus challenge. Soluble neuraminidase, produced in a baculovirus system, consisted of tetramers, dimers and monomers. Only the tetramers were enzymatically active. The immunogenicity decreased very considerably in the order tetra $>$ di $>$ mono. Therefore, we fused the head part of the neuraminidase gene to a tetramerizing leucine zipper sequence; the resulting product was enzymatically active, tetrameric neuraminidase. The protective immunity induced by this engineered neuraminidase, however, remained fairly strain-specific. A third influenza A virus protein, the M2 protein, has only 23 amino acids exposed on the outer membrane surface. This extracellular part, M2e, has been remarkably conserved in all human influenza A strains since 1933. By fusing the M2e sequence to hepatitis B virus core protein, we could obtain highly immunogenic particles that induced complete, strain-independent, long-lasting protection in mice against a lethal viral challenge. Native M2 is a tetrameric protein and this conformation of the M2e part can also be mimicked by fusing this sequence to a tetramerizing leucine zipper. The potential of the resulting protein as a vaccine candidate remains to be evaluated.
\end{abstract}

Keywords: influenza; neuraminidase; M2 protein; hepatitis B virus core; universal vaccine

\section{INTRODUCTION}

The high variability of the antigenic properties of the major influenza surface glycoproteins haemagglutinin and neuraminidase has been a major obstacle to controlling the viral disease by vaccination. We have shown before by nucleotide sequencing of viral genes that 'drift' corresponds to a limited number of mostly single nucleotide changes (Min Jou et al. 1980; Verhoeyen et al. 1980). These results confirmed, at the genetic level, previous data obtained by partial amino-acid determination and by serology. Furthermore, we found that the nucleotide sequence of the human influenza A/Aichi/68 haemagglutinin gene closely resembled the corresponding gene sequence derived from A/duck/Ukraine/63 (Fang et al. 1981). As the former corresponded to an early isolate of the $1968 \mathrm{H} 3 \mathrm{~N} 2$ pandemic, these results provided almost proof that a 'shift' phenomenon is due to the introduction of a gene derived from the animal reservoir in a human virus strain.

We then started to explore whether this basic genetic information could help in the development of improved influenza vaccines. Immunization of mice with purified, recombinant, secreted haemagglutinin indeed provided complete protection against a lethal challenge with mouse-adapted virus (Vanlandschoot et al. 1993; Saelens et al. 1999). But this was only true when the homologous virus strain was used as a challenge, and various attempts to broaden the specificity of protection largely failed. Nevertheless, it may be noted that such recombinant vaccines, made in lower eukaryotes, offer many

*Author for correspondence (fiers@dmb.rug.ac.be). additional advantages, such as cost, the possibility of rapidly adapting to new virus strain specifications, independence from uncertain supplies (e.g. embryonated eggs), etc.

\section{NEURAMINIDASE-BASED VACCINE}

The second immunodominant glycoprotein on the viral surface is the tetrameric neuraminidase. Although in a strict sense antibodies against neuraminidase are not neutralizing, they interfere with spreading of the virus and prevent morbidity in vivo (Johansson et al. 1993). As the gene drifts independently from that of the haemagglutinin (Kilbourne et al. 1990), a neuraminidase-based vaccine can be valuable either as such or in combination with a classical haemagglutinin-based vaccine.

Neuraminidase is a type II protein. Hence, in order to obtain a secreted, recombinant product, we replaced the $\mathrm{N}$-terminal, transmembrane coding part by the haemagglutinin type I signal sequence information (Deroo et al. 1996; Martinet et al. 1997). Immunization of mice with the purified product conferred complete protection against a potentially lethal viral challenge and this protection was transferable by serum. However, efficient protection was only obtained against a challenge virus bearing the homologous subtype neuraminidase. We tried to enhance the response against conserved parts of the molecule by mutating immunodominant epitopes, but were unable to substantially broaden the specificity (Martinet et al. 1998).

Soluble, recombinant neuraminidase, for example produced in a baculovirus/ $S f 9$ cell system, is a mixture of tetrameric, dimeric and small amounts of monomeric 


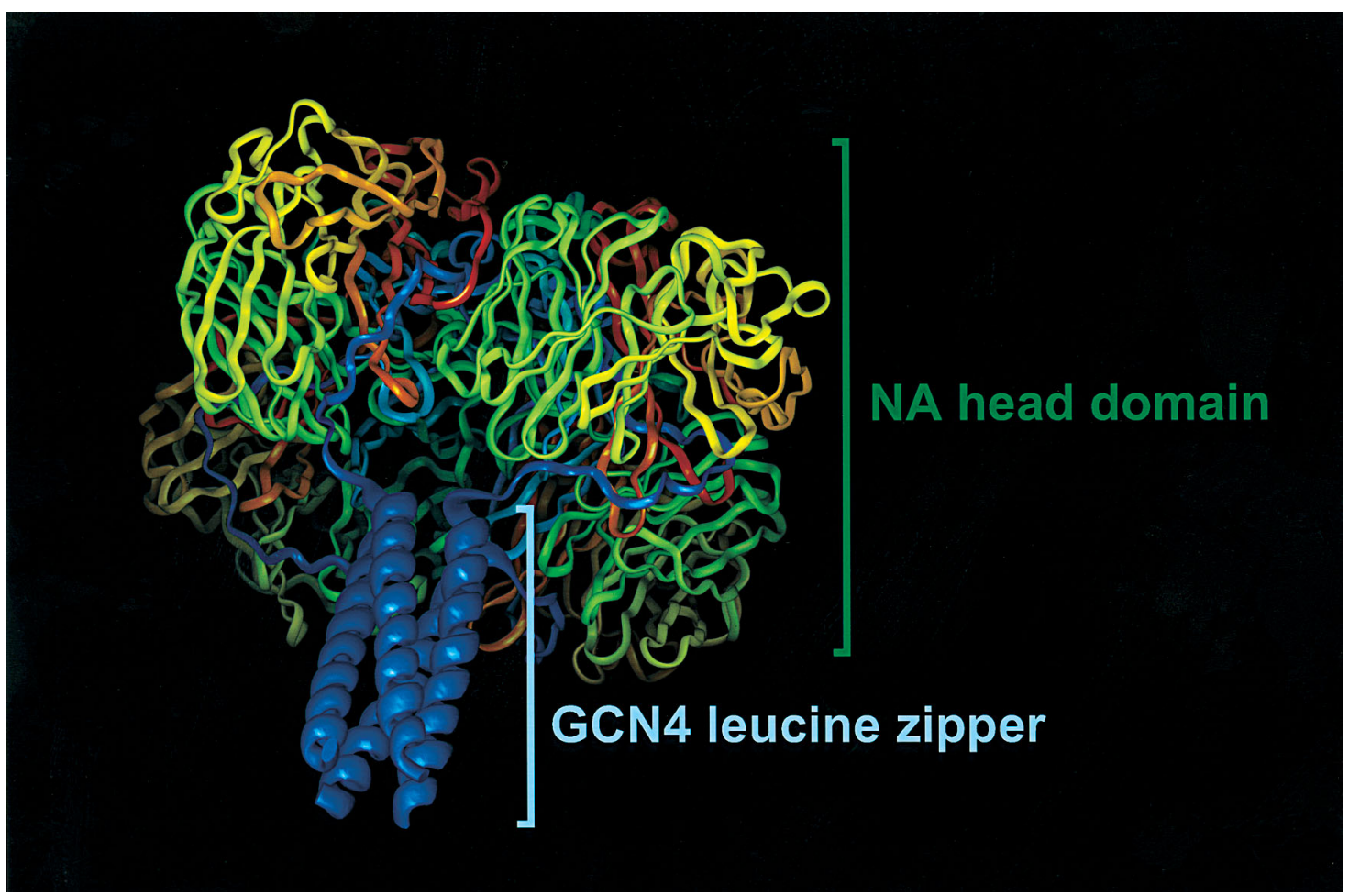

Figure 1. Presumed three-dimensional structure of the chimeric, tetrameric leucine zipper GCN4-neuraminidase based on the known structure of the two constituent proteins.

molecules. Only the first is endowed with enzymatic activity (Deroo et al. 1996). On an equal-weight basis, the tetramers were remarkably more antigenic than the dimers, which in turn were considerably better than the monomers. Therefore, we constructed chimeric molecules consisting of the neuraminidase gene (minus the transmembrane part) fused to a tetramer-inducing sequence such as the leucine zipper domain of the yeast transcription factor GCN4 (Harbury et al. 1993; T. Deroo, W. Min Jou and W. Fiers, unpublished data). The resulting product (figure 1) was indeed exclusively tetrameric and fully enzymatically active, and constitutes an attractive material for a neuraminidase-based vaccine.

\section{M2-BASED VACCINE}

M2 protein is the third virus-coded, integral membrane protein. The tetrameric protein is sparingly present on the virus particles, but is abundant on virusinfected cells, where it functions as a selective ion channel (Lamb et al. 1985; Zebedee \& Lamb 1988; Pinto et al. 1992). Drugs like amantadine or rimantadine target the M2 ion channel and inhibit productive infection (Belshe et al. 1988). Unlike haemagglutinin and neuraminidase, the extracellular part of the M2 protein (M2e) is not subjected to severe immune selection pressure. Indeed, since the first human virus isolates in 1933, hardly any change has occurred in the 23-amino-acid-long M2e sequence (figure 2), except for an aspartate to glycine mutation in $\mathrm{A} / \mathrm{PR} / 8 / 34$ at position 21 , and an additional glycine to glutamate at position 16 in some far-eastern Asian isolates in the 1994-1995 period. M2e is only weakly immunogenic but it has been shown that a monoclonal antibody directed against M2e inhibits influenza virus replication in mice (Treanor et al. 1990).
Hepatitis B virus (HBV) core particles are easy to produce in Escherichia coli and can be readily modified at several positions without loss of their particle-forming properties (Pumpens \& Grens 1999). The icosahedral particles consist of 180 or 240 subunits, which are arranged as dimers (Wynne et al. 1999). These particles are highly efficient in presenting epitopes to the immune system. We have explored the potential of chimeric M2e-HBV core particles as a universal vaccine against influenza A (Neirynck et al. 1999). Immunization was either intraperitoneal together with a mild adjuvant or intranasal without any adjuvant. Both procedures led to appreciable serum antibody titres and provided full protection against a challenge with a lethal dose of a mouse-adapted virus preparation. The protection was long-lasting (half a year in mice) and was based on circulating antibodies, as shown by passive immunization. Most importantly, M2e-HBV could elicit an immune response in mice that protected against a challenge with a different subtype virus. Based on the conservation of the amino-acid sequence of M2e, one may conclude that the protective immunity is universal for all human influenza A strains (the single mutation at position 21 was shown to be irrelevant; the effect of the mutation at position 16 remains to be tested). There are many ways one can explore to improve the antigenicity of the M2e-based vaccine: e.g. one or more copies of M2e can be fused at the $\mathrm{N}$-terminus or inserted internally so that it becomes exposed at the tip of the HBV core spikes; the protaminelike C-terminal region of the HBV core can be replaced by several influenza T-cell epitopes, etc. Also, considering that native M2 protein is a tetramer, one can fuse M2e to the tetramerizing GCN4 leucine zipper sequence or other appropriate multimeric carriers. A number of these possibilities are now being tested. 
virus strain (subtype) 2

A/WS/33 (H1N1)

A/WSN/33 (H1N1)

A/PR/8/34 (H1N1)

A/Fort Warren/1/50 (H1N1)

A/USSR/90/77 (H1N1)

A/Singapore/1/57 (H2N2)

A/Leningrad/134/57 (H2N2)

A/Ann Arbor/6/60 (H2N2)

A/Udorn/72 (H3N2)

A/Port Chalmers/1/73 (H3N2)

A/Bangkok/1/79 (H3N2)

A/NY/83 (H3N2)

A/Guangdong/39/89 (H3N2)

A/Akita/1/94 (H3N2)

A/Hebei/19/95 (H3N2)

A/Niigata/137/96 (H3N2)

A/Shiga/25/97 (H3N2)
$\begin{array}{lllllllllllllllllllllll}S & L & L & T & E & V & E & T & P & I & R & N & E & W & G & C & R & C & N & D & S & S & D\end{array}$

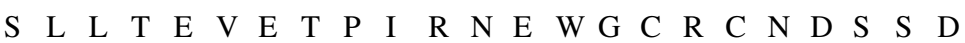

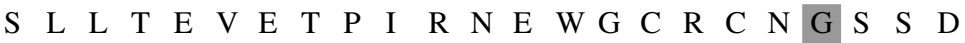

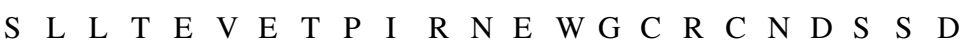

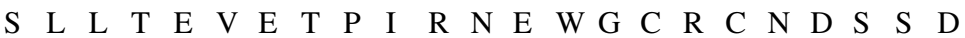

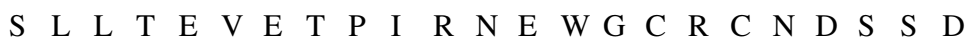

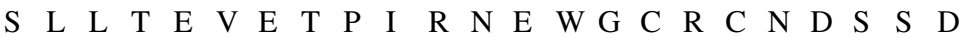

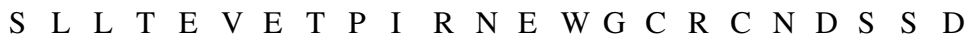

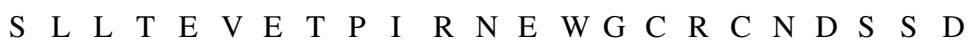

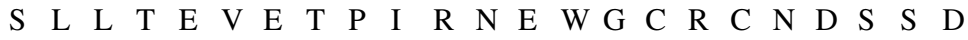

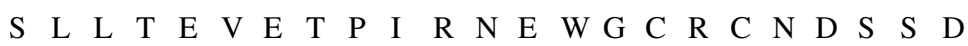

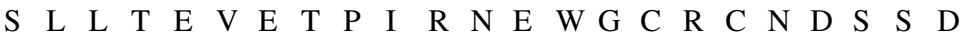

$\begin{array}{lllllllllllllllllllllll}S & L & L & T & E & V & E & T & P & I & R & N & E & W & G & C & R & C & N & D & S & S & D\end{array}$

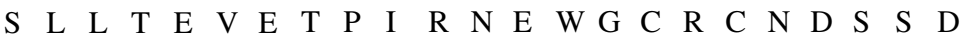

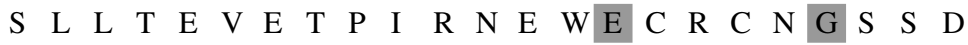

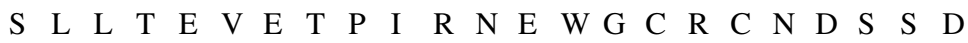

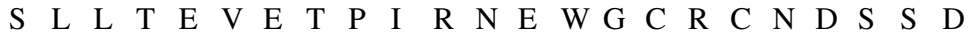

Figure 2. Representative examples of the extracellular amino-acid sequence of M2 proteins since the first human influenza A isolate in 1933. (These data are a subset of a much more extensive compilation; courtesy of Dr M. De Filette (Laboratory of Molecular Biology, Ghent, Belgium).)

\section{REFERENCES}

Belshe, R. B., Smith, M. H., Hall, C. B., Betts, R. \& Hay, A. J. 1988 Genetic basis of resistance to rimantadine emerging during treatment of influenza virus infection. 7. Virol. 62, $1508-1512$.

Deroo, T., Min Jou, W. \& Fiers, W. 1996 Recombinant neuraminidase vaccine protects against lethal influenza. Vaccine 14, 561-569.

Fang, R., Min Jou, W., Huylebroeck, D., Devos, R. \& Fiers, W. 1981 Complete structure of A/duck/Ukraine/63 influenza hemagglutinin gene: animal virus as progenitor of human $\mathrm{H} 3$ Hong Kong 1968 influenza hemagglutinin. Cell 25, 315-323.

Harbury, P. B., Zhang, T., Kim, P. S. \& Alber, T. 1993 Switch between two-, three-, and four-stranded coiled coils in GCN4 leucine zipper mutants. Science 262, 1401-1407.

Johansson, B. E., Grajower, B. \& Kilbourne, E. D. 1993 Infection-permissive immunization with influenza virus neuraminidase prevents weight loss in infected mice. Vaccine 11, 1037-1041.

Kilbourne, E. D., Johansson, B. E. \& Grajower, B. 1990 Independent and disparate evolution in nature of influenza A virus hemagglutinin and neuraminidase glycoproteins. Proc. Natl Acad. Sci. USA 87, 786-790.

Lamb, R. A., Zebedee, S. L. \& Richardson, C. D. 1985 Influenza virus M2 protein is an integral membrane protein expressed on the infected-cell surface. Cell 40, 627-633.

Martinet, W., Saelens, X., Deroo, T., Neirynck, S., Contreras, R., Min Jou, W. \& Fiers, W. 1997 Protection of mice against a lethal influenza challenge by immunization with yeast-derived recombinant influenza neuraminidase. Eur. 7. Biochem. 247, 332-338.

Martinet, W., Deroo, T., Saelens, X., Beirnaert, E., Vanlandschoot, P., Contreras, R., Fiers, W. \& MinJou, W. 1998 Evaluation of recombinant A/Victoria/3/75 (H3N2) influenza neuraminidase mutants as potential broad-spectrum subunit vaccines against influenza A. Arch.Virol. 143, 2011-2019.
Min Jou, W. (and 10 others) 1980 Complete structure of the hemagglutinin gene from the human influenza A/Victoria/3/75 (H3N2) strain as determined from cloned DNA. Cell 19, 683696.

Neirynck, S., Deroo, T., Saelens, X., Vanlandschoot, P., Min Jou, W. \& Fiers, W. 1999 A universal influenza A vaccine based on the extracellular domain of the M2 protein. Nature Med. 5, 1157-1163.

Pinto, L. H., Holsinger, L. J. \& Lamb, R. A. 1992 Influenza virus M2 protein has ion channel activity. Cell 69, 517-528.

Pumpens, P. \& Grens, E. 1999 Hepatitis B core particles as a universal display model: a structure-function basis for development. FEBS Lett. 442, 1-6.

Saelens, X., Vanlandschoot, P., Martinet, W., Maras, M., Neirynck, S., Contreras, R., Fiers, W. \& Min Jou, W. 1999 Protection of mice against a lethal influenza virus challenge after immunization with yeast-derived secreted influenza virus hemagglutinin. Eur. F. Biochem. 260, 166-175.

Treanor, J. J., Tierney, E. L., Zebedee, S. L., Lamb, R. A. \& Murphy, B. R. 1990 Passively transferred monoclonal antibody to the M2 protein inhibits influenza A virus replication in mice. F. Virol. 64, 1375-1377.

Vanlandschoot, P., Maertens, G., Min Jou, W. \& Fiers, W. 1993 Recombinant secreted haemagglutinin protects mice against a lethal challenge of influenza virus. Vaccine 11, 1185-1187.

Verhoeyen, M., Fang, R., Min Jou, W., Devos, R., Huylebroeck, D., Saman, E. \& Fiers, W. 1980 Antigenic drift between the haemagglutinin of the Hong Kong influenza strains $\mathrm{A} /$ Aichi $/ 2 / 68$ and $\mathrm{A} /$ Victoria/3/75. Nature 286, $771-776$

Wynne, S. A., Crowther, R. A., \& Leslie A. G. 1999 The crystal structure of the human hepatitis B virus capsid. Mol. Cells 3, 771-780.

Zebedee, S. L. \& Lamb, R. A. 1988 Influenza A virus M2 protein: monoclonal antibody restriction of virus growth and detection of M2 in virions. F. Virol. 62, 2762-2772. 